International Journal of Modern Physics E

(C) World Scientific Publishing Company

\title{
A statistical model analysis of $K / \pi$ fluctuations in heavy ion collisions
}

\author{
Giorgio Torrieri \\ Theoretical Physics, J.W. Goethe Universitat, Frankfurt A.M., Germany \\ torrieri@fias.uni-frankfurt.de
}

Received 22 February 2007

\begin{abstract}
We briefly describe two statistical hadronization models, based respectively on the presence and absence of light quark chemical equilibrium, used to analyze particle yields in heavy ion collisions. We then try to distinguish between these models using $K / \pi$ fluctuations data. We find that while the non-equilibrium model provides an acceptable description of fluctuations at top SPS and RHIC energies, both models considerably under-estimate fluctuations at low SPS energies.
\end{abstract}

\section{Introduction}

The exploration of the thermal properties of strongly interacting matter, specifically, of its equation of state, transport coefficients, degree of equilibration, phase structure, and the dependence of these on the energy and system size is one of the main objectives of heavy ion research. Thus, it is natural to try to characterize the soft observables in these collisions using statistical mechanics techniques.

While such an approach has a long and illustrious history [1/21314, the systematic and quantitative comparison of data to the statistical hadronization (SH) model is a comparatively recent field. A consensus has developed $5|6| 7|8| 9$ that the SH model can indeed fit most, if not all particle yields measured at experiments conducted at a wide range of energies. Measurements conducted at the GSI Schwerionen Synchrotron (SIS), BNL's Alternating Gradient Synchrotron (AGS),CERN's Super Proton Synchrotron (SPS), and BNL's Relativistic Heavy Ion Collider (RHIC) have successfully been analyzed using SH ansatze.

However, the applied SH models differ regarding the chemical equilibration condition that is presumed. As a result, it has not as yet been possible to agree statistical physics, if any, is responsible for the striking trends observed in the energy dependence of some observed hadronic yields.

In the SH model there are two types of chemical equilibrium: all models assume relative chemical equilibrium 9 , but some also assume absolute chemical equilibrium which implies the presence of just the right abundances of valance up, down, and strange quark pairs. If the system of produced hadrons is considered to be in absolute chemical equilibrium, then at highest heavy ion reaction energy one obtains 

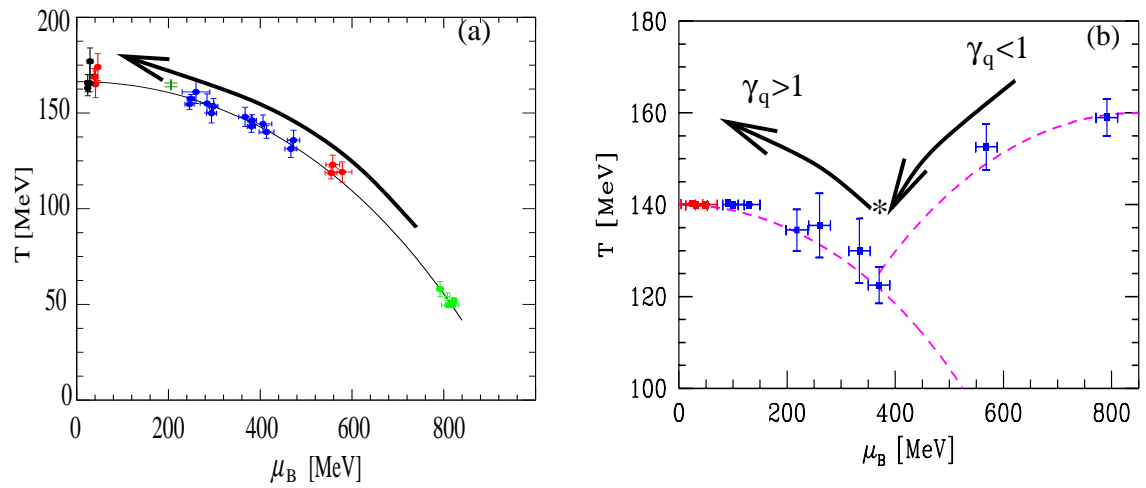

Fig. 1. (Color online) Dependence of freeze-out temperature $T$ and baryo-chemical potential $\mu_{\mathrm{B}}$ on reaction energy in the Equilibrium (a), and non-equilibrium (b) freeze-out models. The direction of the arrow corresponds to increasing $\sqrt{s}$. The equilibrium dependence of $T$ and $\mu_{B}$ in the panel (a) is not significantly altered by the introduction of the fitted phase space occupancy $\gamma_{s}$ and/or the implementation of the Canonical ensemble for strangeness. The "star" in panel (b) corresponds to the point where the transition to the supercooled regime occurs and the phase space changes from chemically under-saturated $\left(\gamma_{q}<1\right)$ to chemically over-saturated $\left(\gamma_{q}>1\right)$. This point also corresponds to the energy of the "kink" and the tip of the "horn"

chemical freeze-out temperature $T \sim 160-170 \mathrm{MeV}$. Values as low as $T \sim 50 \mathrm{MeV}$ are reported at lowest reaction energies available.

The energy dependence of the freeze-out temperature than follows the trend indicated in panel (a) of figure 1 as the collision energy increases, the freeze-out temperature increases and the baryonic density (here baryonic chemical potential $\mu_{\mathrm{B}}$ ) decreases 7 . An increase of freeze-out temperature with $\sqrt{s}$ is expected on general grounds, since with increasing reaction energy a greater fraction of the energy is carried by mesons created in the collision, rather than pre-existing baryons 4 .

Further refinements to the equilibrium model, such as the introduction of the chemical non-equilibrium parameter $\gamma_{s}[9 \mid 10$ and a canonical description of the system at small energies/system sizes 10 do not materially alter the behaviour of temperature and chemical potential shown in the panel (a) of Fig. 1.

When assuming chemical equilibrium, the variation in the freeze-out parameters $T$ and $\mu_{B}$ with energy is remarkably smooth. However, there are non-continuous features in the energy dependence of hadronic observables, such as the "kink" in the multiplicity per number of participants and the "horn" $11|12| 8$ in certain particle yield ratios (top panel of Fig. 2). An effort was made to interpret this in terms of a shift from baryon to meson dominance 7 of the hadron yields. However, in the chemical equilibrium model even the simple observable like $K^{+} / \pi^{+}$remains a smooth function of reaction energy, in contrast to the experimental results (top panel of Fig. (2). Introduction of $\gamma_{s}$ and deviations from the thermodynamic limit, while they help in bringing some of the model predictions closer to the data, has so far not managed to reproduce the sharpness of features such as the kink and the 
horn 10 .

Non-monotonic behaviour of particle yield ratios could indicate a novel reaction mechanism, e.g. onset of the deconfinement phase 12. In such a situation, the smoothness of the chemical freeze-out temperature dependence on energy would be surprising, since it would imply that at all energies, from about $1 A \mathrm{GeV}$ at SIS, to the highest RHIC values, there is no change in either the fireball evolution dynamics, nor any other imprint from the deconfined phase on the freeze-out condition, which, however is visible in the strangeness and entropy yield that $K^{+}$and, respectively, $\pi^{+}$represent. In particular, if the expanding system undergoes a fast conversion from a Quark Gluon Plasma (QGP) to hadrons, chemical non-equilibrium and super-cooling 9 can be motivated by entropy and flavor conservation requirements.

If one abandons the hypothesis of absolute chemical equilibrium, and fits phase space occupancies for both light and strange flavors, the systematic behaviour of $T$ with energy becomes quite different 8 , as is shown in panel (b) of figure 1. The two higher $T$ values at right are for 20 (lowest SPS) and (most to right) $11.6 \mathrm{~A}$ $\mathrm{GeV}$ (highest AGS) reactions. In these two cases the source of particles is a hot chemically under-saturated $(T \sim 170 \mathrm{MeV})$ fireball. Such a system could be a conventional hadron gas fireball that had not the time to chemically equilibrate.

Following the thick arrow in panel (b) of figure 1 we note that somewhat smaller temperatures are found with further increasing heavy ion reaction energies. Here it is possible 9 to match the entropy of the emerging hadrons with that of a system of nearly massless partons when one considers supercooling to $T \sim 140 \mathrm{MeV}$, while both light and strange quark phase space in the hadron stage acquire significant over-saturation with the phase space occupancy $\gamma_{q=u, d}>1$ and at higher energy also $\gamma_{s}>1$. A drastic change in the non-equilibrium condition occurs near $30 \mathrm{~A}$ $\mathrm{GeV}$, corresponding to the dip point on right in panel (b) of the figure 1 (marked by an asterisk). At heavy ion reaction energy below (i.e. to right in panel (b) of figure 11) of this point, hadrons have not reached chemical equilibrium, while at this point, as well as, at heavy ion reaction energy above (i.e. at and to left in panel (b) of figure 1), hadrons emerge from a much denser and chemically more saturated system, as would be expected were QGP formed at and above $30 \mathrm{~A} \mathrm{GeV}$. This is also the heavy ion reaction energy corresponding to the "kink", which tracks the QGP's entropy density (higher w.r.t. a hadron gas), and the peak of the "horn" 11, which tracks the strangeness over entropy ratio (also higher w.r.t. a hadron gas).

Distinguishing the two models described here by yields and particle ratios data has proven to be problematic. While fits with $\gamma_{q}$ consistently achieve a higher statistical significance 8 , the statistical significance of equilibrium models is well above the conventionally accepted minimum of $5 \%$ at all energies, so it can not be argued that the non-equilibrium model is "objectively better" at describing data. Since the two models are physically different, however, it is also not appropriate to just assume the equilibrium model holds because of it's greater "simplicity" (number of parameters). Rather, observables should be found where the two models 
give different predictions. In the next section we will show that fluctuations of $K / \pi$ are an example of such observable.

\section{2. $K / \pi$ Fluctuations in chemical (non)equilibrium}

As shown in 13, fluctuations can be an invaluable test of light quark chemical non-equilibrium. This is because the scaled variance $\sigma_{N}=\left\langle(\Delta N)^{2}\right\rangle /\langle N\rangle$ scales in a different way from $\langle N\rangle$ w.r.t. temperature and the light quark phase space occupancy $\gamma_{q}$.

An increase in $T$, in general, lowers $\sigma_{N}$ because of the greater contribution of resonances that introduce particle correlations. An increase in $\gamma_{q}$, on the other hand, increases $\sigma_{N}$ where $N$ is a number carried by $\pi$ (such as the multiplicity or the electric charge), since the increased quantum (Bose-Einstein) contributions to pion yields are enhanced by the phase space over-saturation specific to $\gamma_{q}$.

Fluctuations are in general more sensitive than yields to detector acceptance cuts. In addition, not understood effects, such as event-by-event volume fluctuations can give significant contributions to the observables that are difficult to describe within statistical models. The solution to second objection, which also resolves part of the first, is to use "dynamical" fluctuations of particle ratios

$$
\sigma_{d y n}^{N 1 / N 2}=\sqrt{\sigma_{N 1 / N 2}^{2}-\left(\sigma_{N 1 / N 2}^{s t a t}\right)^{2}}
$$

where $\sigma_{\text {stat }}$ is the fluctuation in mixed events, that, having no resonance or quantum corrections, should be simply given by Poissonian scaling. $\sigma_{N 1 / N 2}$ contains such correlation terms, so

$$
\sigma_{N 1 / N 2}^{2}=\frac{\sigma_{N 1}^{2}}{\left\langle N_{1}\right\rangle}+\frac{\sigma_{N 2}^{2}}{\left\langle N_{2}\right\rangle}-2 \alpha \frac{\left\langle\Delta N_{1} \Delta N_{2}\right\rangle}{\left\langle N_{1}\right\rangle\left\langle N_{2}\right\rangle}
$$

where the correlation term arises due to resonances decaying into $N_{1}$ and $N_{2}$ (and in general has to be weighted by an experimental reconstruction probability $\alpha$ ). We refer the reader to 14 for details on how to compute the $\sigma$ s described here.

We have used the ratios and yields in the data-samples of $\frac{8}{\text { to }}$ fit $T, \mu_{B, s}, \gamma_{s}$, and the reaction volume, in the model where $\gamma_{q}$ is fitted (non-equilibrium) and where $\gamma_{q}=1$ (chemical equilibrium). The results, together with SPS 15 and RHIC 16 experimental data are shown in Fig. 2, both for the equilibrium model (squares) and the non-equilibrium model (circles). We have also shown the two extreme cases of experimental acceptance, completely preserving the correlations $(\alpha=1$, filled symbols) and completely destroying them ( $\alpha=0$,empty symbols). The effect of the correlations is not negligible, but does not alter the energy dependence of the fluctuations in both models.

As can be seen, $\sigma_{d y n}^{K / \pi}$ at RHIC and the highest SPS energies is compatible with the non-equilibrium statistical model. Fluctuations at lower energies, however, can not be described by any set of chemical parameters that also describes yields. As expected from it's smooth variation of parameters, and smallness of quantum 


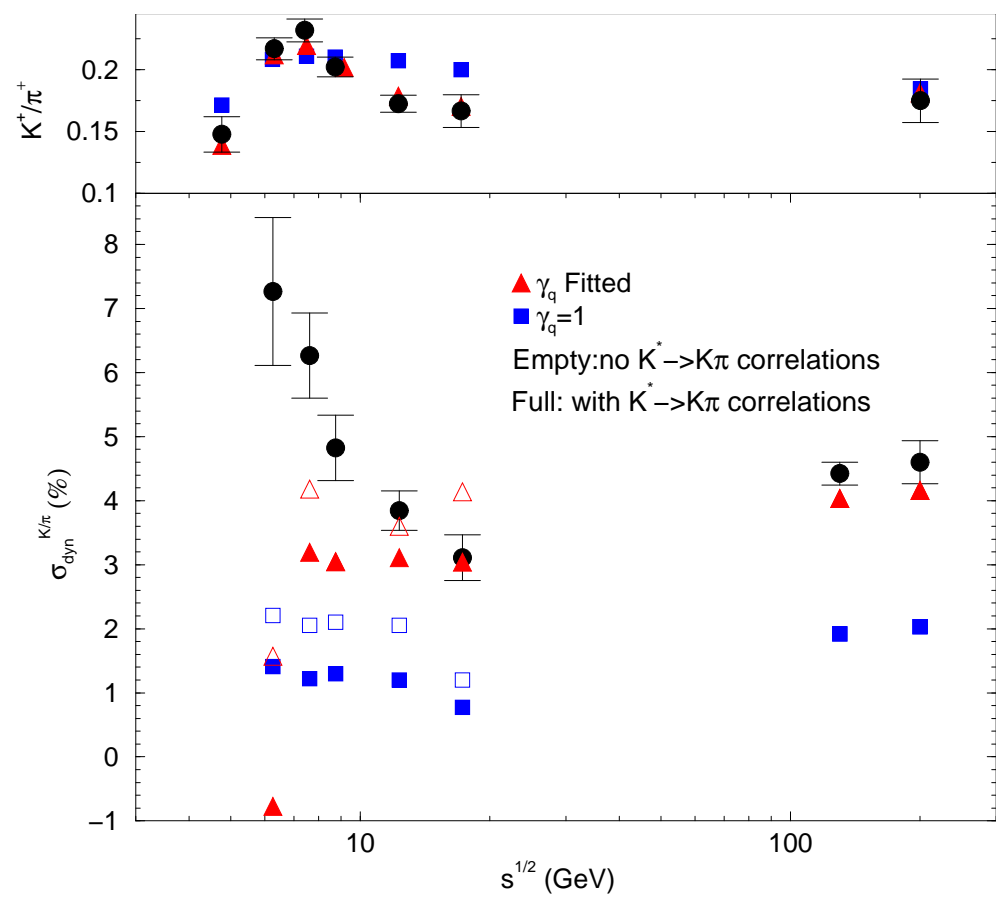

Fig. 2. (Color online) $K / \pi$ fluctuations with model calculations. Top panels shows the ratio dependence on energy

corrections, $\sigma_{d y n}^{K / \pi}$ does not show a substantial variation in the equilibrium $\left(\gamma_{q}=1\right)$ model. This model under-estimates fluctuations at all energies under consideration.

It should be noted that these results, based on preliminary data, should be taken with a measure of caution. The acceptance region of NA49 is highly non-trivial ( $\alpha$ is likely to be neither 0 nor 1 , and strongly momentum-dependant), and our model does not take this into account. While the use of dynamical fluctuations eliminates a some of the dependence of the results acceptance cuts, the acceptance dependence on correlations (for instance, $K^{*} \rightarrow K \pi$ ) remains, and introduces a systematic error in our model. For RHIC results, $\sigma_{K^{ \pm} / \pi^{ \pm}}$, this correlation is negligible since lower-lying resonances do not produce like-sign particles in their decays. For SPS results, $\sigma_{\left(K^{+}+K^{-}\right) /\left(\pi^{+}+\pi^{-}\right)}$, the correlation term is non-negligible but not dominant $\left(\sim K^{*} / K \sim 10 \%\right)$, as can also be seen from the compatibility of top energy SPS and RHIC fluctuations.

For $p / \pi$ fluctuations the greater contribution of resonance correlations $\left(\Delta, \Lambda, \Sigma, \Sigma^{*}\right.$ and $\Xi$ s all decay into $p \pi$, and form $\sim 70 \%$ of the proton yields at all $\sqrt{s}$ ), makes the statistical description of these fluctuations much less reliable without the inclusion of acceptance cuts. For this reason, we do not use this fluctuation in our analysis, beyond noting that both the equilibrium and non-equilibrium 
models severely over-estimate it 17. We await the measurement of this fluctuation at RHIC, whose detectors have a very different acceptance region than SPS's NA49, to fully ascertain the role of limited acceptance in this fluctuation's measured value.

We can only speculate about the reason for why fluctuations at low SPS energies are so above the statistical model estimate. Initial conditions have now been found, at RHIC, to be highly inhomogeneous. if the system produced in heavy ion collisions is perfectly thermalized throughout it's evolution ("a perfect fluid"), than the only trace these inhomogeneities leave at freeze-out is a fluctuation in the system volume, that is cancelled event by event when fluctuations of ratios are considered.

In a system that is not perfectly equilibrated, however, "kinetic" fluctuations due to the random nature of each collision between the system's degrees of freedom can indeed arise. Perhaps this is the origin of the larger $K / \pi$ fluctuations observed at lower SPS energies, either due to a greater impact of the hadronic "corona", or lack of equilibration in the whole system.

To test this speculation, it is necessary to model $K / \pi$ fluctuations through kinetic models that include the fluctuation in the initial condition, "dynamical" fluctuations due to the finite number of dynamical processes that generate Kaons and $\pi \mathrm{s}$ (as well as the microscopic randomness of each process) and a model of the detector's acceptance cuts (as mentioned, highly non-trivial for NA49). Such a study goes beyond the scope of this work.

In conclusion, we have given a description of the two statistical models currently on the market. As a way to differentiate between these models, we have

calculated the $\sigma_{K / \pi}^{d y n}$ as a function of energy, and compared the model predictions to experimental data. The results, while interesting, are not conclusive: The equilibrium model under-estimates $\sigma_{d y n}^{K / \pi}$ at all energies, while the non-equilibrium model describes the higher energies SPS and RHIC acceptably. It however considerably under-estimates $\sigma_{d y n}^{K / \pi}$ at lower SPS energies. While this might be an indication that the system at these energies is not completely thermalized, and hence the random nature of each microscopic interaction enhances the final fluctuations beyond the statistical expectation, this can not for now be conclusively established.

The author thanks the Von Humboldt foundation for the financial support given, Frankfurt University for the hospitality provided, as well as the QM2006 conference organizers for the financial support that allowed him to attend the conference. We also thank Michael Hauer, Mark Gorenstein, Marek Gazdzicki, Marcus Bleicher, Volker Koch, Sangyong Jeon, and Johann Rafelski for stimulating discussions.

\section{References}

1. E. Fermi Prog. Theor. Phys. 5, 570 (1950).

2. I. Pomeranchuk Proc. USSR Academy of Sciences (in Russian) 43, 889 (1951).

3. LD Landau, Izv. Akad. Nauk Ser. Fiz. 17 51-64 (1953).

4. R. Hagedorn R Suppl. Nuovo Cimento 2, 147 (1965).

5. P. Braun-Munzinger, D. Magestro, K. Redlich and J. Stachel, Phys. Lett. B 518, 41 (2001) arXiv:hep-ph/0105229. 
6. P. Braun-Munzinger, I. Heppe and J. Stachel, Phys. Lett. B 465, 15 (1999) arXiv:nucl-th/9903010.

7. J. Cleymans, H. Oeschler, K. Redlich and S. Wheaton, arXiv:hep-ph/0607164.

8. J. Letessier and J. Rafelski, Eur. Phys. J. A 29, 107 (2006)

9. Letessier J, Rafelski J (2002), Hadrons quark - gluon plasma, Cambridge Monogr. Part. Phys. Nucl. Phys. Cosmol. 18, 1, and references therein

10. F. Becattini et. al., Phys. Rev. C 69, 024905 (2004)

11. P. Seyboth et al. [NA49 Collaboration], Acta Phys. Polon. B 36, 565 (2005),

12. M. Gazdzicki and M. I. Gorenstein, Acta Phys. Polon. B 30, 2705 (1999)

13. G. Torrieri, S. Jeon and J. Rafelski, Phys. Rev. C 74, 024901 (2006) arXiv:nucl-th/0503026.

14. G. Torrieri, S. Jeon, J. Letessier and J. Rafelski, Comput. Phys. Commun. 175, 635 (2006)

15. M. Gazdzicki, J. Phys. Conf. Ser. 27, 154 (2005) arXiv:nucl-ex/0507017.

16. Das S [STAR Collaboration] (2005), Event by event fluctuation in $\mathrm{K} / \mathrm{pi}$ ratio at RHIC, arXiv:nucl-ex/0503023

17. G. Torrieri, arXiv:nucl-th/0702020 